\title{
Kirigami artificial muscles with complex biologically inspired morphologies
}

\author{
Sina Sareh, Jonathan Rossiter \\ Department of Engineering Mathematics and Bristol Robotics Laboratory, \\ University of Bristol, Bristol BS8 1TR, UK \\ E-mail: S.Sareh@bristol.ac.uk
}

\begin{abstract}
In this paper we present bio-inspired smart structures which exploit the actuation of flexible ionic polymer composites and the kirigami design principle. Kirigami design is used to convert planar actuators into active 3D structures capable of large out-of-plane displacement and that replicate biological mechanisms. Here we present the burstbot, a fluid control and propulsion mechanism based on the atrioventricular cuspid valve, and the vortibot, a spiral actuator based on Vorticella campanula, a ciliate protozoa. Models derived from biological counterparts are used as a platform for design optimisation and actuator performance measurement. The symmetric and asymmetric fluid interactions of the burstbot are investigated and the effectiveness in fluid transport applications is demonstrated. The vortibot actuator is geometrically optimised as a camera positioner capable of 360 degree scanning. Experimental results for a one-turn spiral actuator show complex actuation derived from a single degree of freedom control signal.
\end{abstract}

\section{Introduction}

Biological systems are capable of functioning in real world environments that have high degrees of variability. Typically featuring a high degree of controlled flexibility in their bodies, they are highly adaptable and interact safely with each other and with the environment. These properties are highly attractive for new robotic systems where the soft-soft interface predominant in biology is intrinsically safer than the traditional hard-hard interface found in the majority of engineering systems (Sanan 2011). Many complex mobility and manipulation mechanisms in nature are difficult or costly to replicate in conventional rigid robotics (Ilievski et al 2011). Hence, designing bio-inspired robotic systems that exploit the compliant, elastic and flexible properties of biological mechanisms is a major focus of smart materials research (Bar-Cohen et al 2007; Kim and Tadokoro 2007). In this paper we present a new approach to designing, optimising and fabricating 3D bio-inspired robotic actuator components using the principle of kirigami (Badalucco 2000). We show how kirigami is used to create two new robotic systems: the Burstbot, based on the atrioventrical cuspid valve; and the Voritbot, based on the Vorticella campanula protozoa.

Nature has provided a rich source of inspiration for robotic scientist wishing to design flexible robots. The patterns and mechanisms of movement in particular are significant features of microorganisms, animals, and plants and hence a major focus of bio-inspired and bio-mimetic engineering. These include, for example; Chlamydomonas reinhardtii, a single-celled green alga, swims using two anteriorly inserted whiplash flagella (Silflow and Lefebvre 2001); The powerful contractile mechanism in vorticella mark these spring-like organisms as having one of the fastest biological engines (Mahadevan and Matsudaira 2000); Elephant trunks, octopus arms and mammal tongues are attractive compliant biological mechanisms that exhibit multi-modal extension, bending and twisting motions (Kier and Smith 1990; Wainwright and Bennett 1992; Napadow et al 2002). Long tentacles in Tubastrea (Sun coral) produce asymmetric inflow to catch passing zooplankton to feed the animal 
(Blomquist et al 2006). We can classify the active mechanism in nature into three main categories based on the form of actuation, as summaries in Table 1.

Table1. Classification of actuation forms devised by nature and subsequent bio-inspired engineering systems

\begin{tabular}{lll} 
Actuator type & Examples in nature & Engineering systems \\
\hline Flexible manipulators & $\begin{array}{l}\text { Fluid interactions at sub-millimetre scales by Cilia } \\
\text { and related organelles. }\end{array}$ & $\begin{array}{l}\text { Micro-fluidic flow control } \\
\text { devices. }\end{array}$ \\
$\begin{array}{l}\text { Expandable/deployable } \\
\text { systems }\end{array}$ & $\begin{array}{l}\text { Streaming of protoplasm in Amoeba, } \\
\text { Spasmoneme contraction in Vorticella, } \\
\text { contraction of the cavity in Jelly fish, shape } \\
\text { transformation in human muscles and heart } \\
\text { valves. }\end{array}$ & $\begin{array}{l}\text { Smart clothes, diaphragm and } \\
\text { jet pumps, turbulence } \\
\text { amplifiers and kirigami } \\
\text { artificial muscles }\end{array}$ \\
& $\begin{array}{l}\text { Simultaneous extension, bending and twisting in } \\
\text { mammalian tongues, tentacles, etc. }\end{array}$ & $\begin{array}{l}\text { Multi-modal flexible robotic } \\
\text { manipulators. }\end{array}$
\end{tabular}

\subsection{Electroactive polymers for bioinspired soft robotics}

Soft robotics is an emerging research field employing "soft-smart" technologies to improve versatility, functionality and biological compatibility of future robots. A complete soft robot is an autonomous programmable machine, more closely related to biological organisms than conventional robotics and typically made from soft materials that allow continuous deformations. One such soft-smart material is the class of electroactive polymers (EAPs) (Bar-Cohen et al 2007) that undergo shape change in response to electric stimulation and act rather like natural muscles. Many characteristics of soft EAPs including pliability, scalability, low weight, silent operation, and relatively large actuation strain make them well-suited for robotic applications (Shahinpoor et al 2005; Bar-Cohen et al 2007).

Ionic Polymer Metal Composites (IPMCs) are a promising class of ionic EAP that generate large bending motions under a low driving voltage $(\sim 2 \mathrm{~V})$. A typical IPMC actuator consists of a thin ionic membrane, usually Nafion or Flemion, with gold or platinum electrodes plated on both faces. The morphology of the actuator, structure of its ionic membrane, conductivity of the electrodes and the level of hydration play a major role in their operation characteristics ( $\mathrm{Wu}$ and Nemat-Nasser 2004). The ready fabrication of IPMCs into thin sheets makes then ideally suited for use as kirigami robotic actuators. In this study, we report on modelling, fabrication and experimental results for two kirigami artificial muscle actuators. These single degree of freedom actuators are capable of generating complex biologically-inspired actuation profiles resembling the flexion of the mammalian cuspid valve and the coiled contractions of the vorticella respectively.

\subsection{Kirigami soft robotics}

Kirigami is a variation of origami, Japanese art of paper folding, which includes cutting of the paper. Kirigami methods have attracted considerable interest from mathematicians, roboticists, artists and architects for designing deployable mechanisms. Saito et al 2011 introduced the concept of a cellular kirigami morphing wingbox. Three-dimensional actuators have been developed by transforming programmed two-dimensional structures via bending, twisting, and folding mechanisms (Jeong et al 2011). In this study, a novel design method for soft robots based on kirigami techniques is proposed to reproduce two biologically inspired morphologies. 
Although soft bending actuators such as IPMCs are generally unsuitable for fabrication through origami (folding) methods on their own, they are more amenable to the kirigami design process. We demonstrate a Kirigami modelling approach where flat actuator membranes are selectively cut in order to produce complex three dimensional morphologies.

\section{Burstbot: a soft active structure for flow control inspired by the atrioventrical heart valve}

Here we aim to design an electro-active bimorph structure for flow control, inspired by the structures and motion of the cuspid heart valve and sun corals.

\subsection{Biological inspiration}

Many convergent morphing structures are known in nature, including the cuspid valve in mammals, the convergent 'zipper' structure of Tubastrea (sun coral) (Blomquist et al 2006) and many other sea anemones and corals. The aortic heart valve, lying between the left ventricle and the aorta in mamalian heart, opens to let blood flow from the left pumping chamber to the main body artery (aorta). The cusps of the heart valves are passive structures that flex open when subject to flow in one direction and seal the heart valves when flow is present in the opposite direction. A normal aortic valve has three cusps (a tricuspid valve) and its opening and closing phases are shown in figure 1a.

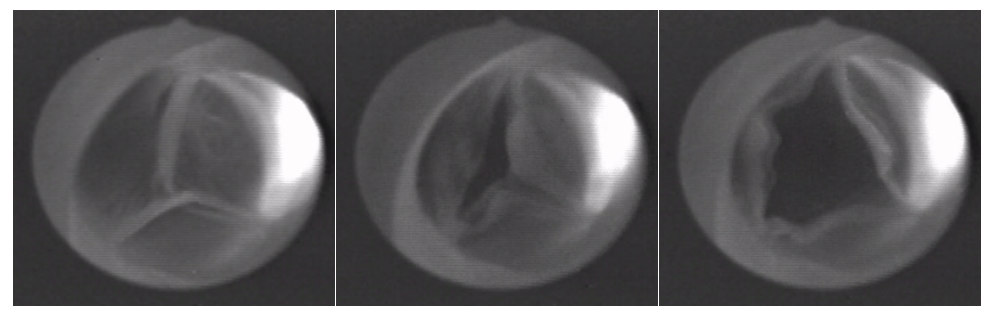

(a)

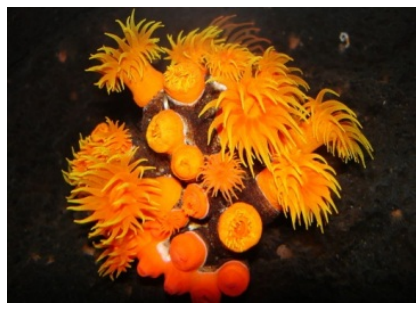

(b)

Figure 1. (a) Opening and closing of the three cuspids of the aortic heart valve [images CC-SA-BY Valveguru], (b) the Tubastrea [image CC-BY Franklin Samir Dattein]

Tubastrea is a genus of coral in the phylum Cnidaria and another example of a convergent zipper structure found in nature, as shown in figure 1b. In general, locomotion and feeding (mass transfer) mechanisms in Cnidaria family are mainly based on jet propulsion and asymmetric flow generation through oral arms. Long tentacles in Tubastrea produce an asymmetric inflow to catch passing zooplankton and hence to feed the animal.

Here, we introduce a novel kirigami multi-cusp bimorph structure using IPMCs inspired by the biological mechanisms in figure 1. In the following, the modelling of the burstbot morphology and potential of the proposed actuator for flow control applications are discussed.

\subsection{Actuator design, Characterisation and modelling}

Starting with a flat sheet of IPMC we use kirigami design principles to create a multi-cuspid bioinspired compliant actuator. Using only a series of $n$ radial cuts of length $S$, we form $n$ identical triangular cusps with common central apex and segment angle $2 \pi / n$, as shown in Figure 2a. When a voltage is applied to the membrane the cusps bend together out of the membrane plane and form complex 3D structures.

Note that the area of each cuspid that bends is a triangle, rather than a complete segment of the circle. For example, for the 8-cuspid actuator in figure 2 a actuation is constrained to the octagon (shown as a 
dashed line) formed by joining the outer ends of all the cuts. It is clear that by increasing the number of segments $n$ the active portion of the enclosed circle $C$ increases, but with a consequent reduction in passive rigidity and increase in actuator complexity. The normalized bendable area (active portion of the central circle) for burst actuators with up to 16 cusps is shown in figure $2 \mathrm{~b}$. A burstbot with 8cusps has active area $90 \%$ of the central circle. Above this number the trend of the graph flattens significantly. In subsequent design and experiments we focus on an 8-cusp burstbot, a number of segments chosen to give a large active area $(90 \%)$ and a relatively low mechanical complexity.

To initially characterise the bending response of a triangular cuspid element a single IPMC cusp was fabricated from $50 \mu \mathrm{m}$ thick ionic membrane (Nafion 112) as shown in figure 3a. The cusp was mounted along the dashed line, a dc voltage was applied and the radius of curvature of the actuator was measured. Figure $3 \mathrm{~b}$ shows voltage:curvature response for the single $45^{\circ}$ cusp where $s=2 \mathrm{~cm}$.

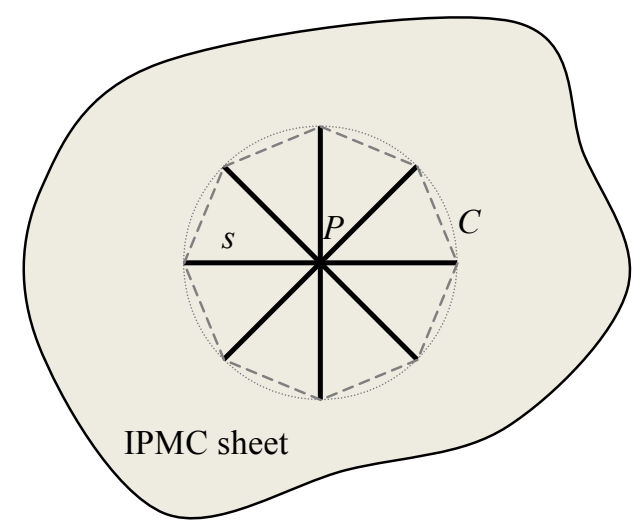

(a)

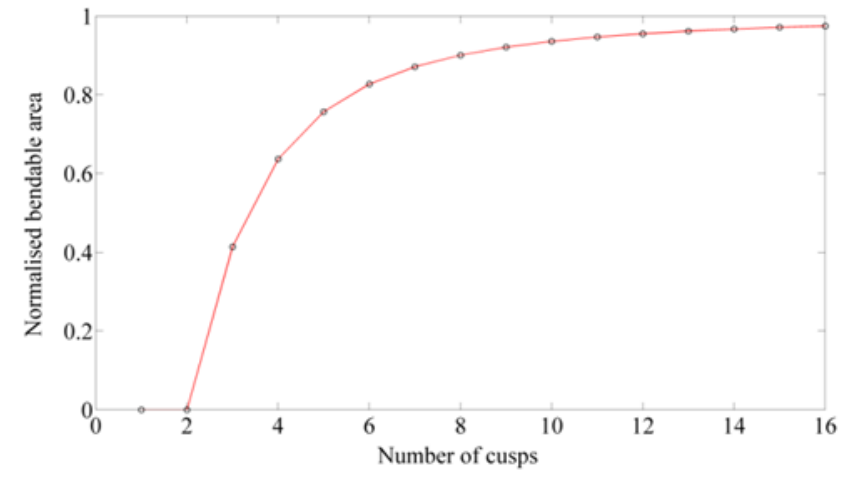

(b)

Figure 2. (a) Example of an 8-cuspid kirigami actuator fabricated by making 8 radial cuts (bold lines) of length $s$ from the central point $P$. (b) Graph of normalized active area vs. the number of cusps.

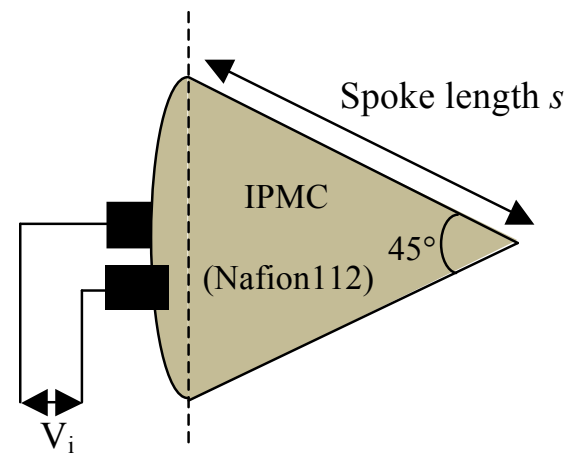

(a)

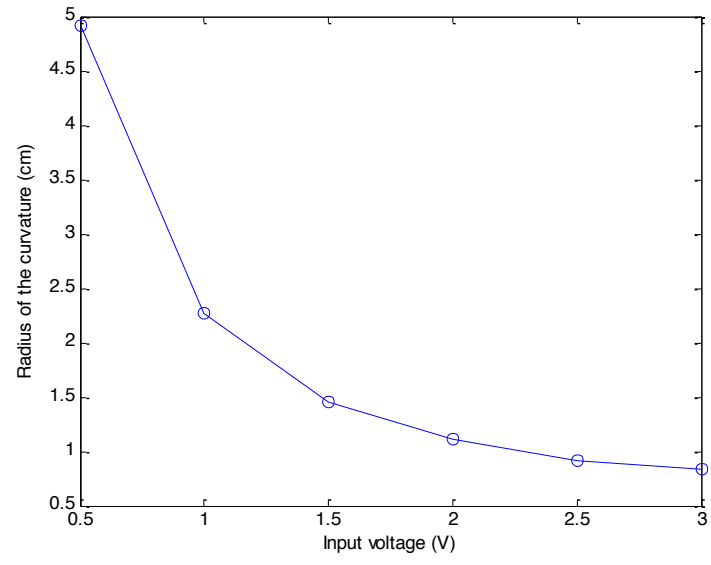

(b)

Figure 3. (a) Characterising a single $45^{\circ}$ cusp. (b) Voltage:curvature relationship

The physical specifications of the aperture are among key design factors for many application of this actuator, for example as a valve; the aperture area associates with the amount of fluid allowed to pass through the hole. To more effectively identify the operating capabilities of the multi-cuspid actuator, a bimorph model of the flexible burst actuation with 8 cusps has been developed. Given the input 
voltage and cusp spoke length we calculate the aperture area and tip-to-tip distance for opposing cusps. We are seeking to design a burst actuator using Nafion 112 IPMC, a very flexible actuator suitable for miniaturisation, and ideally to drive the actuator under $2 \mathrm{~V}$ to avoid high voltage degradation and damage to the material. In the subsequent simulations however we present the results for voltages to $3 \mathrm{~V}$, where some long term damage may occur if used for long periods. Our initial design constraint is for the cuspid tip to be maximally perpendicular to the actuator plane when excited by a voltage up to $2 \mathrm{~V}$. Perpendicular actuation is expected to generate minimal turbulence in fluid control applications, although larger angles of actuation may be desirable in other applications such as mixing.

To simulate the aperture opening, two sets of design parameters have been defined; $S=\left\{\mathrm{s}_{1}, \mathrm{~s}_{2}, \mathrm{~s}_{3}\right\}=$ $\{1,1.5,2\} \mathrm{cm}, \mathrm{V}=\left\{\mathrm{v}_{1}, \ldots, \mathrm{v}_{6}\right\}=\{0.5,1,1.5,2,2.5,3\}$ volt, where $\mathrm{S}$ and $\mathrm{V}$ represent discrete sets for the spoke length and the input voltage respectively. Actuators $\mathrm{D}_{1}, \mathrm{D}_{2}$ and $\mathrm{D}_{3}$ are associated with spoke lengths of $S_{1}, S_{2}$ and $S_{3}$ and are shown in scale in figure 4.

Figure 4 illustrates the side view of two oppositely placed cusps simulated for combinations of $\mathrm{S}$ and $\mathrm{V}$, input data sets. It can be seen that, the bigger actuator produces greater opening for a given voltage and therefore demonstrates a better actuation profile. Table 2 summarises the achievable tip deflection angles for three different sizes of the cusps under prominent excitation voltages $\{1 \mathrm{~V}, 2 \mathrm{~V}, 3 \mathrm{~V}\}$. This shows that the desired tip deflection of around 90 degrees is not achievable for $\mathrm{D}_{1}$, the smallest actuator, even at maximum voltage.
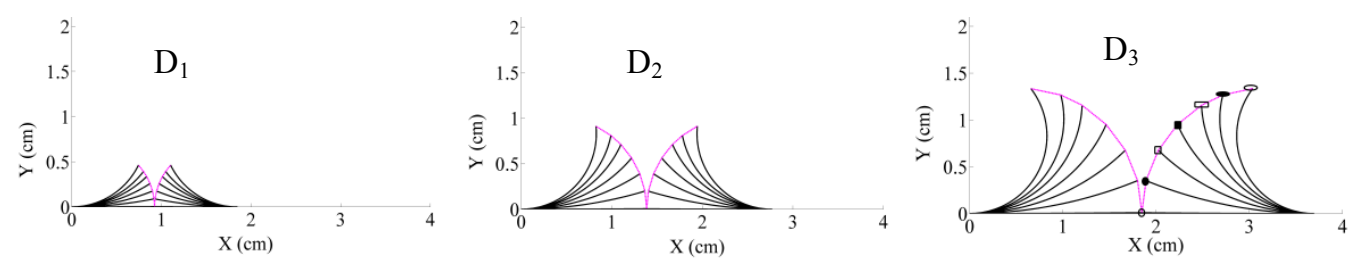

\begin{tabular}{|ll|}
\hline $3 \mathrm{~V}$ & $\circ$ \\
$2.5 \mathrm{~V}$ & - \\
$2 \mathrm{~V}$ & $\square$ \\
$1.5 \mathrm{~V}$ & - \\
$1 \mathrm{~V}$ & $\circ$ \\
$0.5 \mathrm{~V}$ & $\bullet$ \\
0 & $\circ$ \\
\hline
\end{tabular}

Figure 4. Simulation of cuspid actuator, side view at three different sizes, $\mathrm{D}_{1}, \mathrm{D}_{2}$, and $\mathrm{D}_{3}$

However $\mathrm{D}_{2}$ is capable of 95.2 degrees of deflection, but the required input voltage to cause this bending $(3 \mathrm{~V})$, makes it a less suitable choice. $\mathrm{D}_{3}$, the largest actuator, generates 87.2 degrees of tip deflection under safe excitation voltage of $2 \mathrm{~V}$, most closely satisfying the design objectives. Hence, we focus on the simulation and actuation of the $\mathrm{D}_{3}$ actuator in this study.

Table 2. Tip deflection angle of cusps simulated at three different sizes and voltage excitations

\begin{tabular}{ccccc} 
& \multicolumn{3}{c}{ Tip angle $(\mathbf{d e g})$} \\
Actuator ID. & Spoke length $(\mathbf{c m})$ & $\mathbf{1 V}$ & $\mathbf{2 V}$ & $\mathbf{3 V}$ \\
\hline $\mathrm{D}_{1}$ & 1 & 21.2 & 43.6 & 63.5 \\
$\mathrm{D}_{2}$ & 1.5 & 33 & 65.4 & 95.2 \\
$\mathrm{D}_{3}$ & 2 & 43.9 & 87.2 & 126.2
\end{tabular}

The three dimensional morphing model of the Nafion 112 IPMC Bustbot was constructed based on the single cusp bending data in figure $3 \mathrm{~b}$ and the opening aperture was calculated. Figure 5 shows the three dimensional view of a single cuspid $\left(D_{3}\right)$ when excited by a range of voltages. Here we assume zero electrical resistance along the electrodes. 


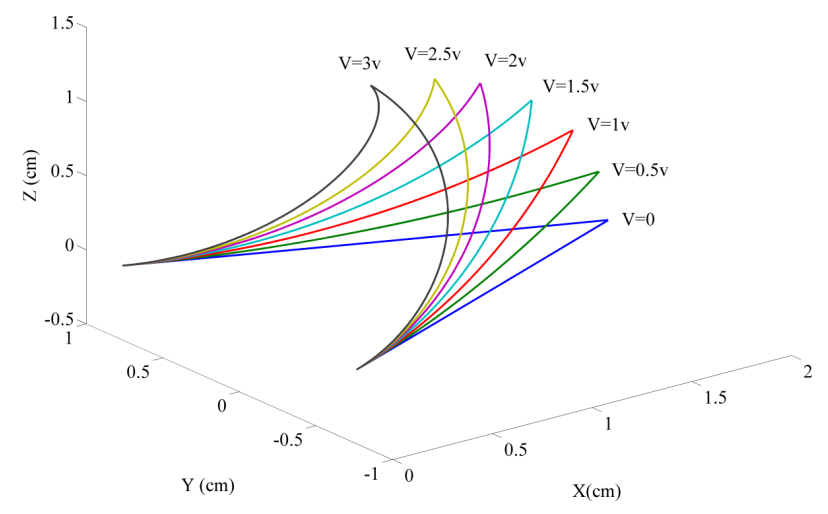

Figure 5. Three dimensional representation of $D_{3}$ actuator for different voltages

Having defined a complete morphing profile for a single cuspid, now we are able to assemble the complete burstbot actuator. An 8-cusp burstbot structure, presented in figure 6, is capable of generating a variety of movement patterns depends on the excitation profile. Figures $6 \mathrm{a}$ and $6 \mathrm{~b}$ illustrate the aperture opening when the actuator is excited under $2 \mathrm{~V}$ and $3 \mathrm{~V}$ respectively. The bottom view of the burstbot aperture under $1 \mathrm{~V}, 2 \mathrm{~V}$ and $3 \mathrm{~V}$ input signals are shown in figure6c.

(a)

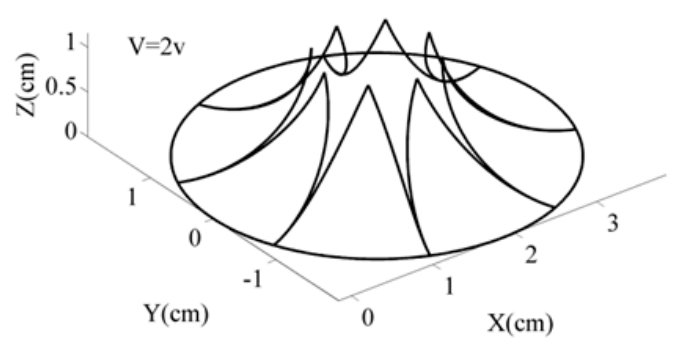

(b)

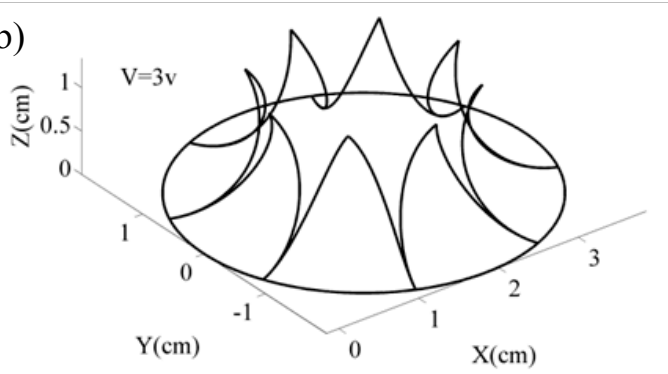

(c)

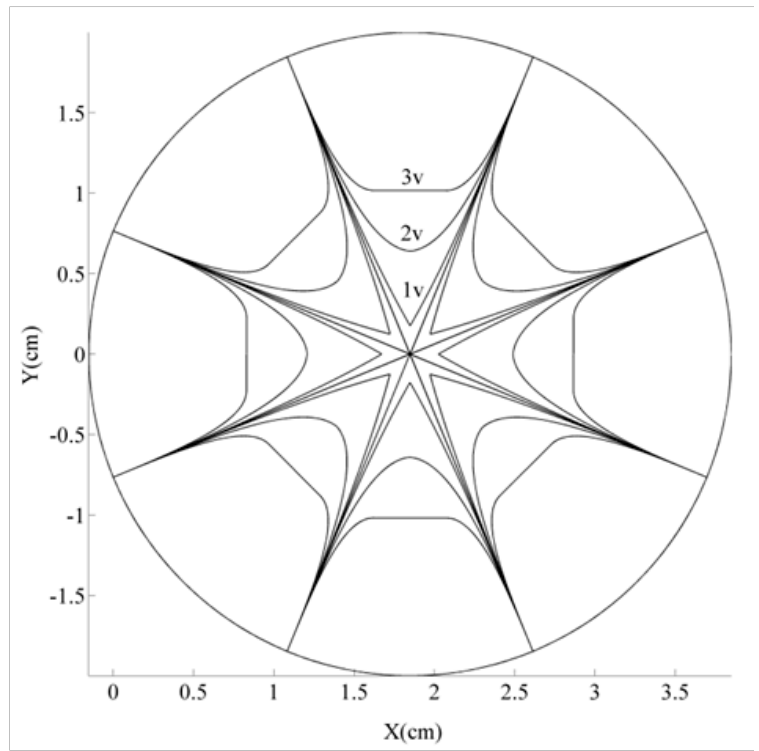

Figure 6. Three dimensional representation of the burstbot $\mathrm{D}_{3}$ at (a) $2 \mathrm{~V}$ and (b) $3 \mathrm{~V}$, and (c) aperture of the burstbot under prominent input voltages

The calculated aperture areas, normalised by the whole circular area of the actuator are shown in figure $7 \mathrm{a}$. This shows the capability of $\mathrm{D}_{3}$ to open to $17 \%$ and $31 \%$ of maximum aperture when exited by $2 \mathrm{~V}$ and $3 \mathrm{~V}$, respectively. Corresponding tip-to-tip distances at these voltages for two oppositely located cusps are $1.3 \mathrm{~cm}$ and $2 \mathrm{~cm}$, as shown in figure $7 \mathrm{~b}$. 


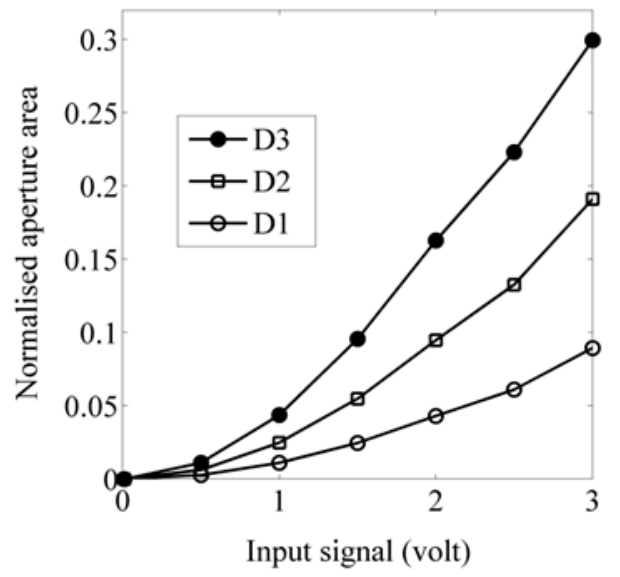

(a)

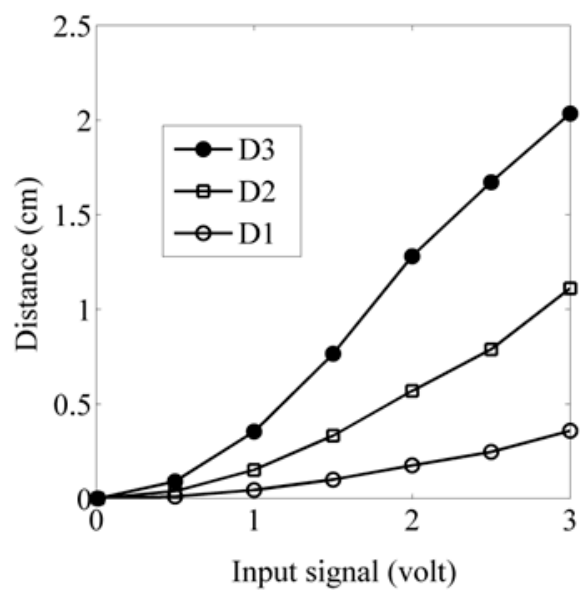

(b)

Figure 7. Simulation of the proposed 8-cuspid burstbot actuator. (a) Normalised 2D aperture area vs. voltage, (b) tip-to-tip distance for opposing pairs of cuspids.

\subsection{Fabrication and testing of burstbot}

A piece of gold-plated $5 \mathrm{~cm} \times 5 \mathrm{~cm}$ Nafion 112 IPMC sheet fabricated using the Oguro-Asaka method of electro-less chemical plating was prepared. The star-like kirigami pattern, shown in figures 2 and 6 , was marked on the IPMC sheet using a soft pencil. To protect contacting electrodes of opposite polarity during the actuation, Electro-discharge Machining (EDM) (Rossiter et al 2011) was used to remove the gold electrodes along the pencil-drawn straight lines of the kirigami pattern before cutting the material using a scalpel. The IPMC sheet was sandwiched between two laser-cut acrylic frames. We connected both sides of the actuator to a Hokuto-Denki HA-151 Potentiostat/Galvanostat, itself driven by a National Instruments PCI-6229 data acquisition device. Circle-shaped copper tape was used to provide good electric conduction on each side of the actuator around the perimeter of the aperture. Front views of the burstbot at rest and when actuated with $2 \mathrm{~V}$ are shown in figure $8 \mathrm{a}$ and figure $8 \mathrm{~b}$, respectively. 


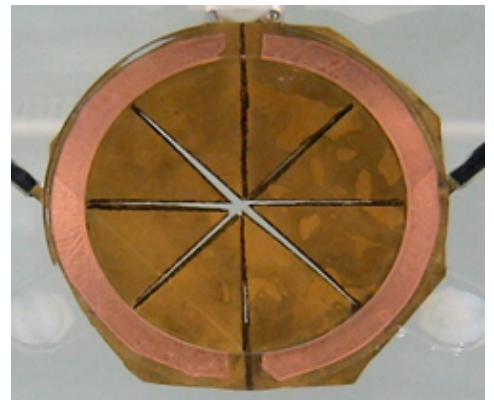

(a)

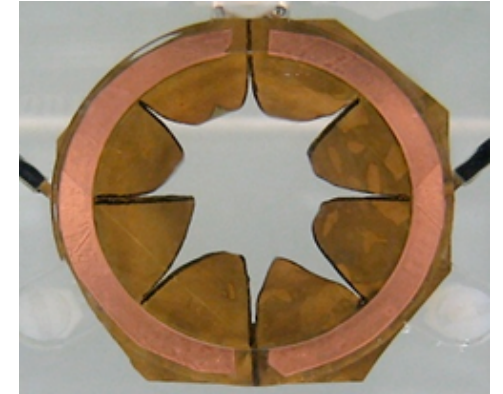

(b)
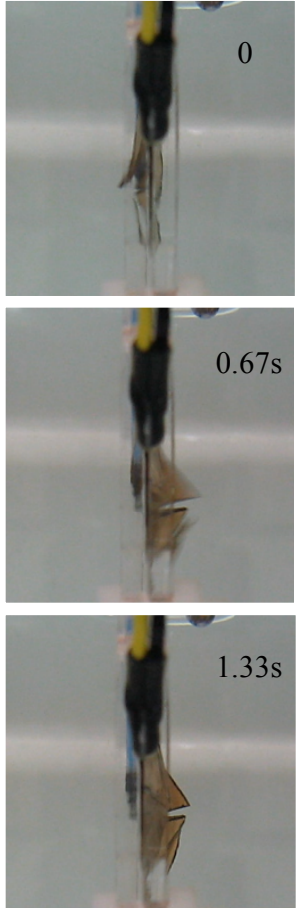
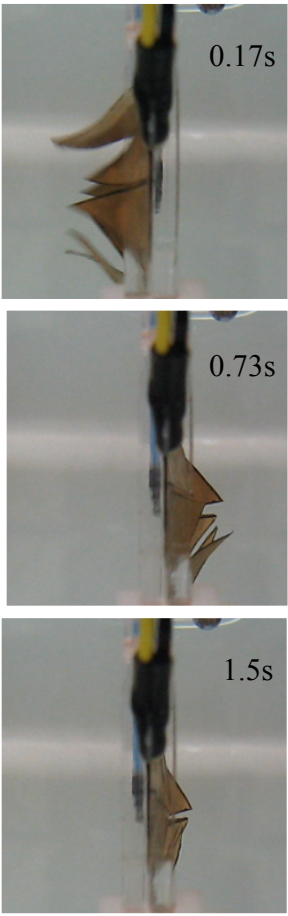
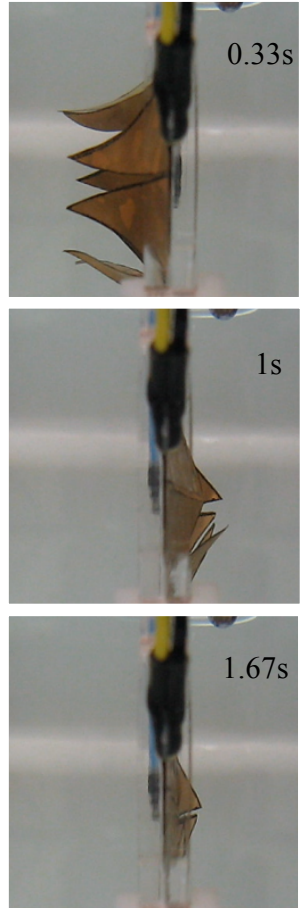

(c)
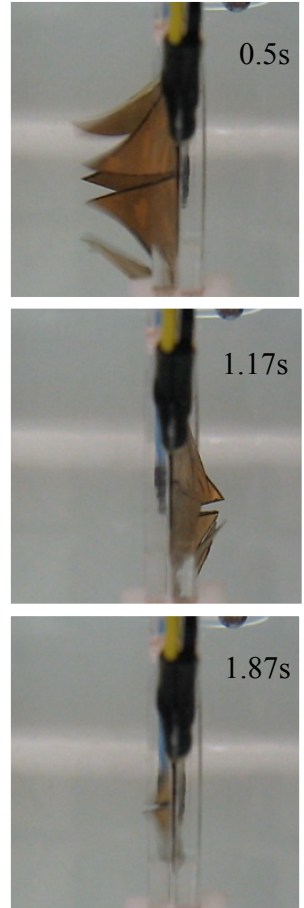

Figure 8. Burstbot before (a) and during (b) excitation at $2 \mathrm{~V}$.

\subsection{Burstbot as an asymmetric fluid flow generator}

In this section, the potential of an IPMC actuator with burstbot morphology for uni-directional flow generation is investigated. Proper activation of the burst structure is essential to the effective operation of the flow generator. For example, if the IPMC actuator is powered using symmetric bipolar excitation signals the generated flow will also be symmetric, resulting in a zero net mass flux jet (Mane et al., 2005). Also, excitation of the actuator under a unipolar input signal results in actuation drift in successive operation cycles (Sareh et al 2010). In the following experiments the Burstbot is therefore driven by an asymmetric charged-balanced bipolar input signal. This enhances the ability of the actuator to produce strong uni-directional fluid flow, whilst ensuring consistency of actuation over time. We drove the burstbot with an asymmetric charge-balanced pulse wave with a duty cycle of $30 \%$ as illustrated in Figure 9a. This signal was applied to the Burstbot and the aperture opening was recorded using a video camera. Figure $9 \mathrm{~b}$ shows the aperture area in response to the input signal in $9 \mathrm{a}$. Note that there is a very large aperture during the application of the positive pulse and a much smaller opening in the opposite direction during the lower negative pulse. In figure $9 \mathrm{~b}$ also the opening and 
closing time of the aperture and the duration required for charge balancing in the first cycle of pumping are expressed by $t_{o 1}, t_{c 1}$ and $t_{b 1}$, respectively. The average opening, closing and balancing time during three cycles of operation are $0.53 \mathrm{sec}, 0.2 \mathrm{sec}$ and $1.27 \mathrm{sec}$, orderly. By defining the balance-excluded actuation time as the summation of opening and closing time in a cycle, a balanceexcluded actuation frequency of $1.37 \mathrm{~Hz}$ is calculated. However, to prevent the actuator from the actuation drift, a low voltage charge balancing is required to be completed in each cycle which results in achieving a the total actuation frequency to $0.5 \mathrm{~Hz}$.

The ability of the Burstbot actuator to generate unidirectional fluid flow was tested by mounting the burstbot vertically in the middle of a large water tank. Concentrated blue dye (methylene blue) was slowly introduced to one side of the actuator as it was stimulated and the movement of the dye was captured by video camera. The input signal in figure 9a was applied, resulting in a large rapid actuation of the cuspids to the left followed by a smaller and slower actuation back to the right. Two tests were undertaken where the dye was introduced either to the left half of the water tank or to the right half. Figure 10a shows percentage of blue dye present on the two sides of the actuator when dye was introduced to the right half of the tank. The amount of the blue dye in any part of the image was calculated by summing the blue components of the RGB image in that area. There is a clear unidirection pumping action as the dye is pushed from the right to the left, ending with an approximately equal amount of dye on both sides of the actuator. Note that the two stages of the pumping action are clearly shown in the undulations in figure $10 \mathrm{a}$, where there is a large positive pumping stroke followed by a smaller relaxation stroke. To confirm that the movement of dye from the right to the left was not due to diffusion the experiment was repeated with dye introduced to the left side only. Figure 10b shows the percentage of blue dye in the two sides as the actuator was stimulated. Note that there is no flow of dye from the left to the right, confirming that the unidirectional pumping action has a far greater effect on dye motion than diffusion.

It is interesting to visualise fluid flow during a full cycle of the actuator. Figure 11a shows the stream of blue dye moving from right to left during the positive part of the control signal. In contrast Figure $11 \mathrm{~b}$ shows a smaller trail of dye being pushed from the left back into the right half. By optimising the charged balanced control signal further we expect to minimise the effect of back relaxation and thus maximise unidirectional fluid flow. Clearly the motion of the biological systems which inspired the burstbot are well suited to their operating environments and further optimisation of the burstbot is required, both in terms of geometry and control signals, to match the particular environment in which it will operate.

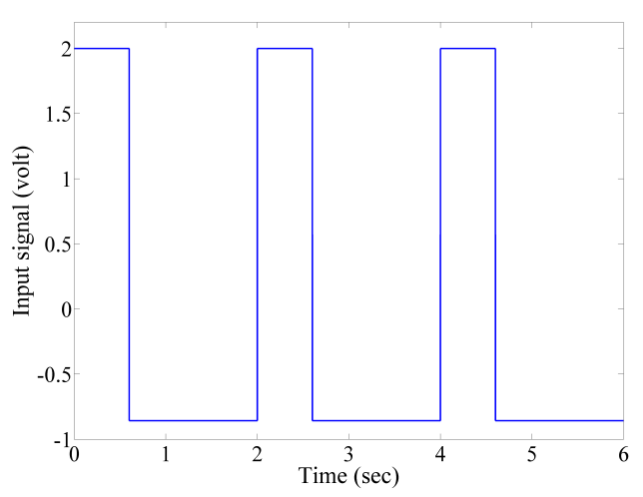

a)

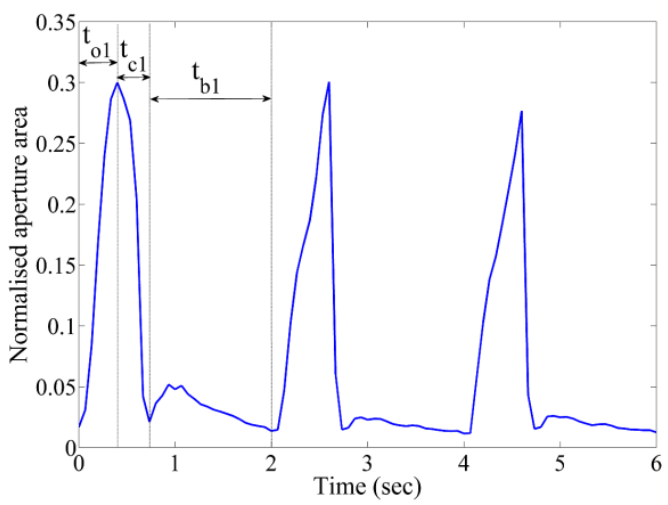

(b)

Figure 9. Input signal and the aperture area profile for three cycles of actuation 


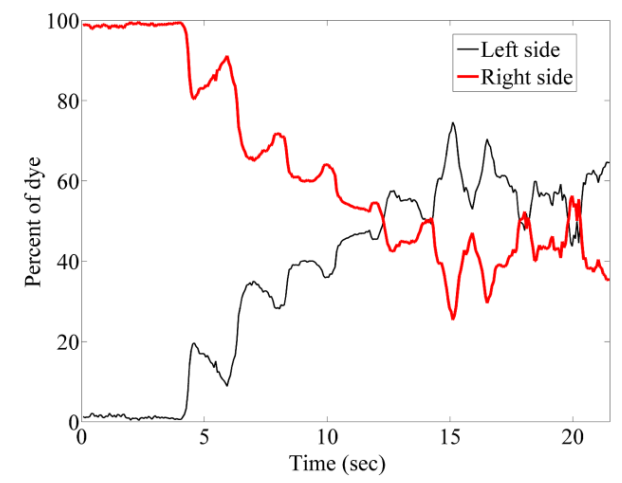

(a)

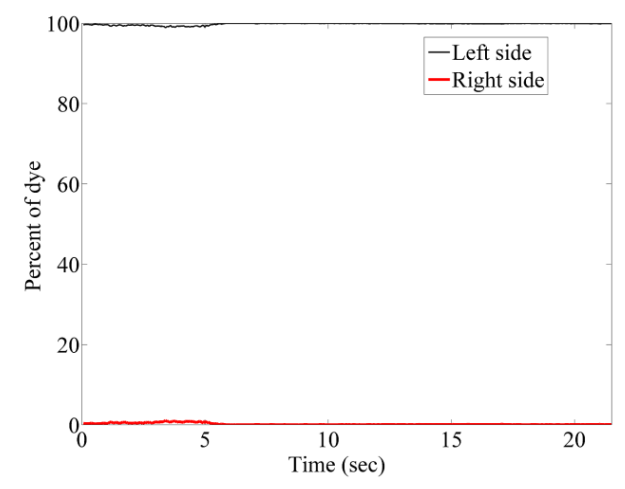

(b)

Figure 10. Movement of dye from right to left (a), and from left to right (b) under postive (right to left) flow generation

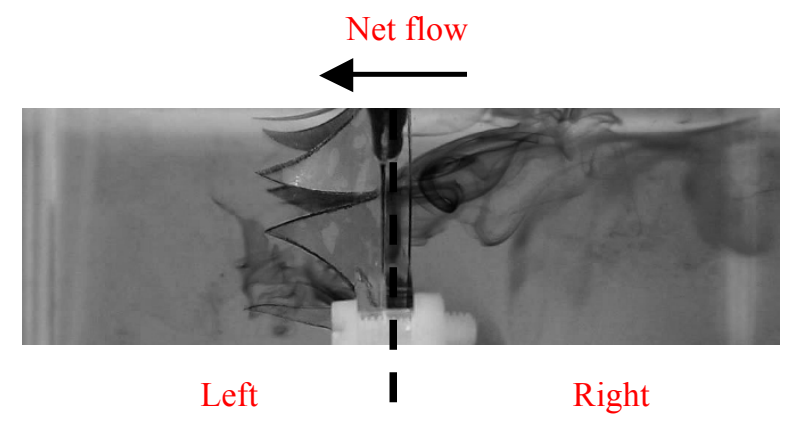

(a)

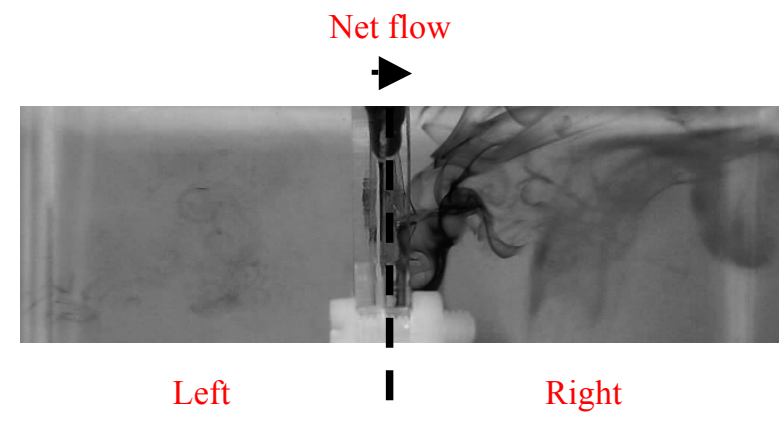

(b)

Figure 11. Movement of dye showing positive flow generation (a), and back relaxation (b).

\section{Vortibot: a robotic analogue to a powerful biological engine}

One of the fastest actuation mechanisms in biology is the linear motor employed in stalked protozoa such as Vorticella campanula. Vorticella consist of a bell-shaped zooid and a slender stalk (100-200 $\mu \mathrm{m}$ in length and $2-3 \mu \mathrm{m}$ in diameter) that is anchored to a substrate (Upadhyaya et al 2008), as shown in figure 12. Actuation takes the form of contractions of the stalk whereby it deforms from a visibly straight filament to a tightly packed spring-like spiral. The spiral contractions of the vorticella are a biological mechanism which is expected to have many uses in engineering and robotic systems from micro-robotic propulsion systems and fluid mixing to novel spiral scanning applications.

Vorticella contract and elongate in response to changes in the concentration of calcium ion. The rapid contraction of the stalk occurs by the release of $\mathrm{Ca}^{2+}$ ions, and this normally takes $7-8 \mathrm{~ms}$. The organelle responsible for the contraction is a contractile fibril, helically bound within an outer elastic sheath, and is known as the spasmoneme. During contraction the spasmoneme shortens, causing the stalk to coil. The stalk returns to the original length within a few seconds when the $\mathrm{Ca}^{2+}$ concentration is reversed (France 2007). Moriyama et al. 1998 estimated that the maximum contracting force in a single vorticella stalk is greater than $55.8 \mathrm{nN}$. 


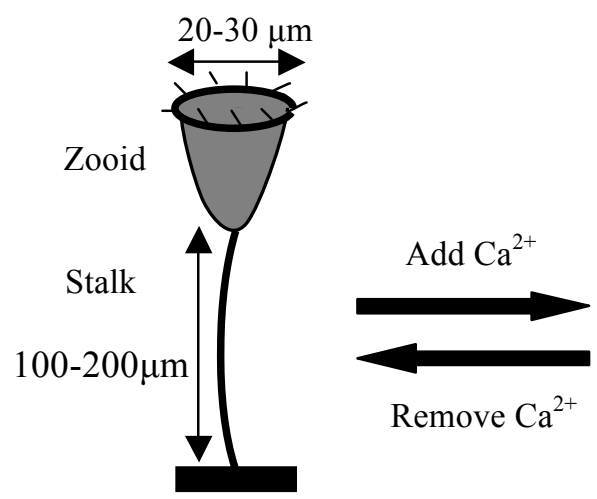

(a)

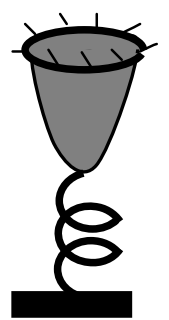

(b)

Figure 12. Illustration of a Vorticella with an (a) extended and (b) contracted spasmoneme

\subsection{Morphologies for a vortibot, the robotic vorticella}

There are several architectures for 3D spiral actuators with potential to reproduce the motion of biological vorticella. We can categorise these based on the principle tangent, normal, and binormal vectors describing their structures. Figure 13 shows four of the simplest helical structures, binormal helix, normal helix, binormal conical helix, and normal conical helix, formed from a flat ribbon actuator such as an IPMC. Of these four structures the binormal helix and binormal conical helix actuate predominantly in the radial axis with smaller actuation in the longitudinal direction. In contrast the normal helix and normal conical helix are more attractive because their prominent actuation is in the longitudinal direction. Unfortunately the stacked spiral structure of the normal helix makes it difficult to fabricate from IPMCs. The intrinsic features of the normal conical helix morphology which can be cut in one piece from a lamina membrane without the need for plastic deformation through heat treatment prevents the spirals from overlapping during the bipolar actuation and hence is ideal for fabrication using IPMCs. It is also important to note that Li et al 2011 introduced a helical IPMC actuator for the radius control of biomedical active stents using the binormal morphology. It is reported that the torsional motion of this actuator is more significant than its longitudinal motion, confirming its low suitability for this study.

We will therefore exploit the normal conical helix in our subsequent modelling, fabrication and testing of an artificial vorticella spasmoneme. Addition benefits of the conical helix include the ability to fabricate it from a single sheet of IPMC actuator material and the mitigation against accidental contact of parts of the IPMCs with different polarity.

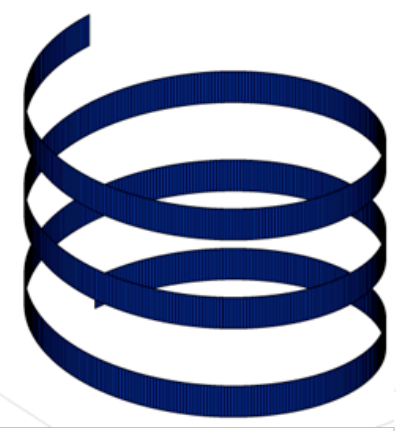

(a)

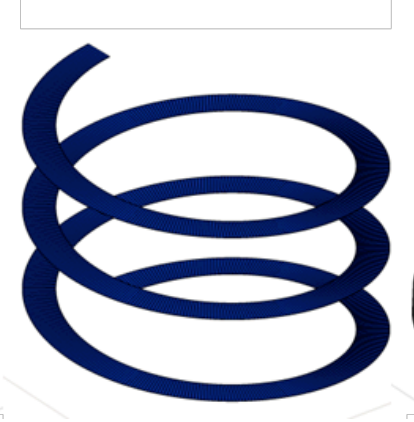

(b)

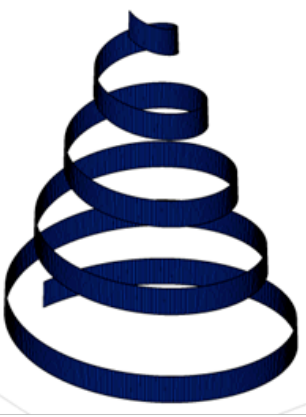

(c)

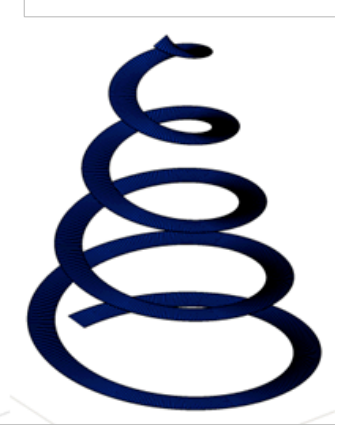

(d)

Figure 13. Helical morphologies; (a) binormal helix, (b) normal helix, (c) binormal conical helix, and (d) normal conical helix. 


\subsection{The conical spiral actuator}

We model the structure of the normal conical helix as essentially a two dimensional Archimedean spiral, as shown in Figure14, which deforms into the third dimension by constant-curvature flexion of the actuator material forming the spiral. Note that in our case we restrict all turns of the spirals to the same width; hence we truncate the Archimedean spiral to remove the first turn extending from the centre where this width would be less than all subsequent turns.

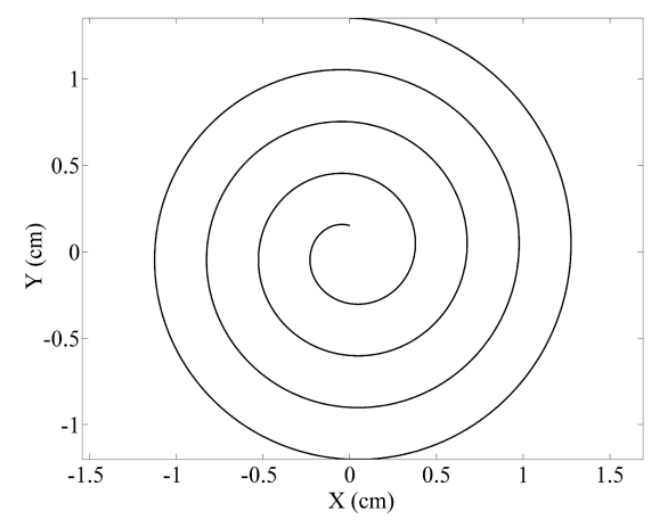

Figure 14. A truncated Archimedean spiral with four turns and $\mathrm{R}_{\mathrm{v}}=0.15 \mathrm{~cm}$

Given geometric model parameters $R_{b}$ and $R_{v}$ denoting the minimum radius of the spiral and the maximum radius respectively, the radius of a zero width spiral coil with $N$ turns is given by $\mathrm{r}(\theta)$,

$$
\mathrm{r}(\theta)=\mathrm{R}_{\mathrm{b}}+\left(\mathrm{R}_{\mathrm{v}}-\mathrm{R}_{\mathrm{b}}\right) \frac{\theta}{2 \pi N}
$$

where, $0<\theta<2 \pi N$

A conic spiral may now be defined as a spiral on a conic surface, with the distance to the apex $h$ (actuator rise) defined as a monotonic function of $\theta$,

$$
\mathrm{Z}(\theta)=h \frac{\theta}{2 \pi N}
$$

\subsection{Maximum actuator rise}

The vertical rise of the spiral actuator can be calibrated with regards to the bending capabilities in IPMC materials. In the following we consider the geometry of bending and the IPMC voltage-radius relationship.

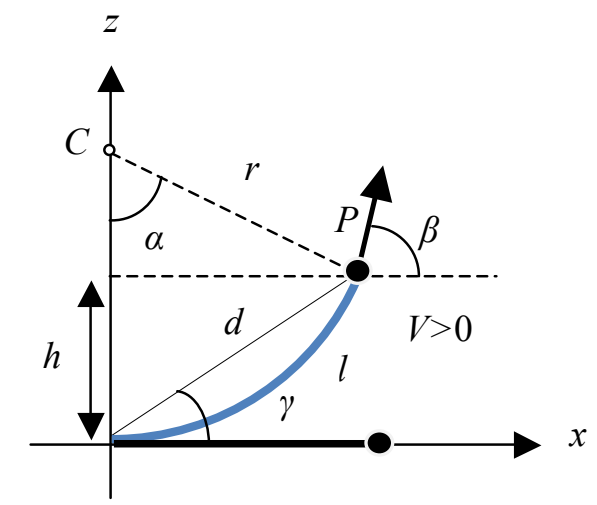

Figure 15. Positive bending of a single constant-curvature actuator of length $l$ in response to voltage $V$ 
The geometry of bending for an idealised IPMC cantilever actuator is illustrated in figure15, where the vertical rise of the actuator,

$$
h=d \sin (\alpha / 2)
$$

and where $\mathrm{d}$ is the chord length,

$$
d=2 r \sin (\alpha / 2)
$$

and hence given $l=r . \alpha$,

$$
h=2 r \sin ^{2}(l / 2 r)
$$

To preserve the motion verticality in the spiral actuator the rise should be strictly increasing; therefore the arc angle $\alpha$ must not exceed 90 degrees, that is $\alpha<\pi / 2$, or equivalently $r>2 l / \pi$. For gold-plated Nafion 115 IPMCs the relationship between radius of curvature and voltage was found from the experimental data to be,

$$
r_{115}=94.09 \mathrm{e}^{-1.30 \mathrm{~V}}
$$

Hence, rewriting (6), for any specific length of the actuator, $l$, a maximum applicable voltage (constrained by $\alpha<\pi / 2$ ) can be expressed as

$$
\mathrm{V}_{115, \max }(l)=0.77 \ln (0.02 l / \pi)
$$

Similarly, gold-plated Nafion 112 also demonstrates an exponential behaviour in its voltage-radius relationship when actuated under input voltages of $1 \mathrm{~V}$ and above. Exploiting this similarity, we used Nolinear Least Squares (NLS) method to extract the transfer function from the experimental data for this thinner IPMC material within the actuation range of $1 \mathrm{~V}$ to $2.5 \mathrm{~V}$ expressed as

$$
r_{112}=8.7 \mathrm{e}^{-0.95 V}
$$

and therefore the maximum applicable voltage can be obtained through

$$
\mathrm{V}_{112, \max }(l)=1.05 \ln (0.23 l / \pi)
$$

\subsection{Generating the IPMC surface}

To grow a two-dimensional cross section for the spiral actuator, a square with the same dimensions as the IPMC cross section, was evolved along the length of the conical helix using Rodrigues' rotation formula. Note that here we only consider bending in line with the principle circular (tangential) direction of the spiral IPMC. The resulting spiral actuator is shown in figure 16.

Considering a width $\mathrm{W}$ for the spiral IPMC strip, the relationship between base and apex radii and the spiral parameters $(\mathrm{W}, \mathrm{N})$ can be expressed as

$$
\mathrm{R}_{\mathrm{v}}=\frac{\mathrm{W}}{2}, \mathrm{R}_{\mathrm{b}}=(\mathrm{N}+0.5) . \mathrm{W}
$$




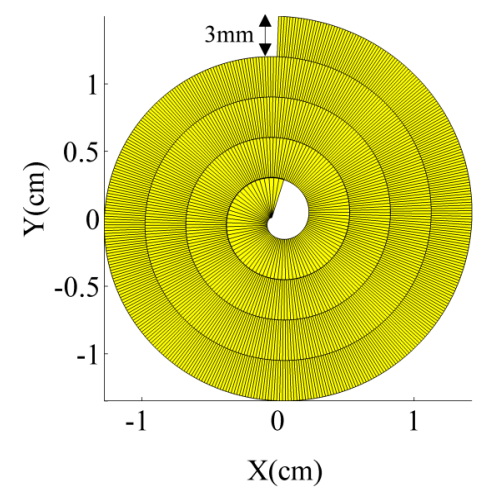

Figure 16. Illustration of a spiral surface with four turns and strip width of $3 \mathrm{~mm}$.

We can now simulate the actuation of a Nafion 112 IPMC Spasmoneme. Let us consider an actuator with a single turn $(\mathrm{N}=1)$ and a strip width of $\mathrm{W}=3 \mathrm{~mm}$. From (9), the maximum applicable voltage is found to be $2.07 \mathrm{~V}$, hence the results of simulation under $1 \mathrm{~V}$ and $2 \mathrm{~V}$ excitations are presented in figure 17.

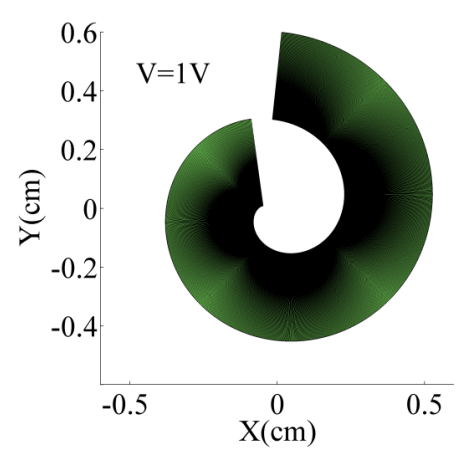

(a)

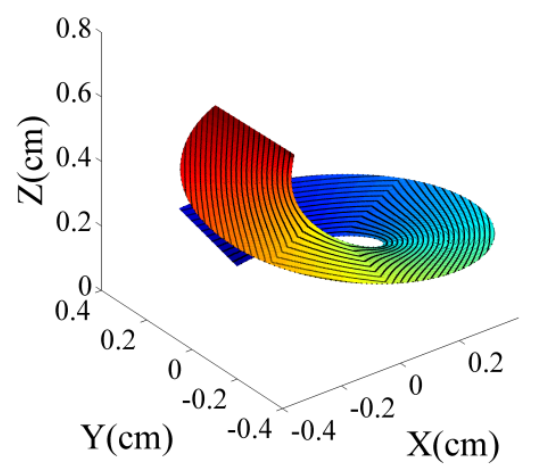

(c)

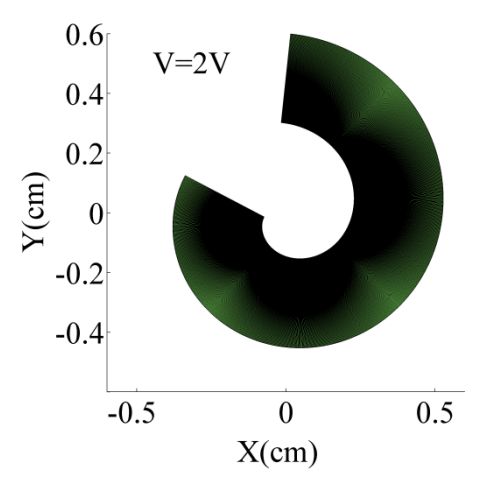

(b)

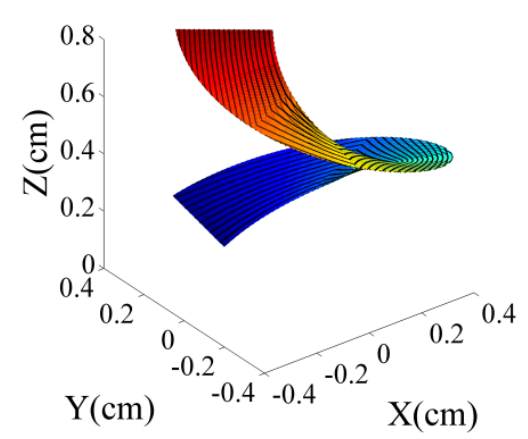

(d)

Figure 17. (a) Top view of the actuator under 1V, (b) top view of the actuator under 2V, (c) three-dimensional view under $1 \mathrm{~V},(\mathrm{~d})$ three-dimensional view under $2 \mathrm{~V}$

A maximum of $0.92 \mathrm{~cm}$ vertical rise for the actuator under $2 \mathrm{~V}$ excitations is expected with reference to the simulation results. To validate the geometrical model, we fabricated an IPMC actuator from 
Nafion 112 IPMC with the same dimensions as figure 17. Figure 18 shows actuation of this IPMC structure when stimulated with $2 \mathrm{~V}$.

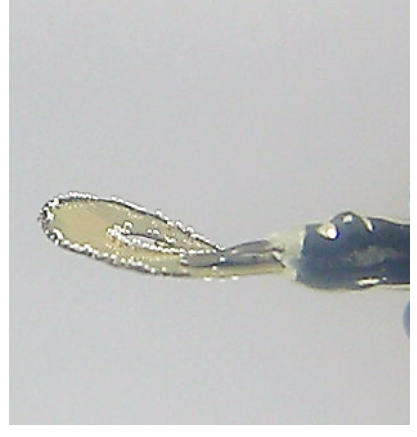

(a)

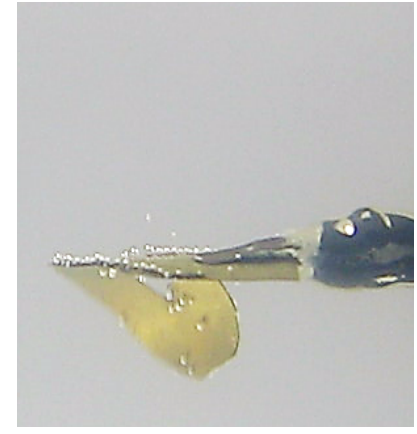

(b)

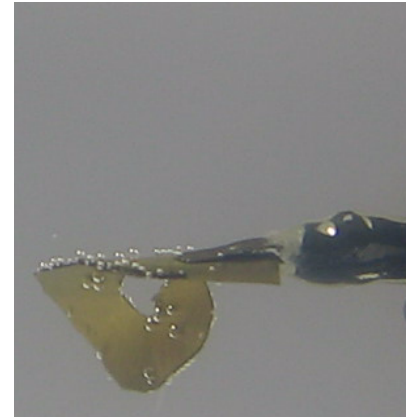

(c)

Figure18. Actuation of a one-turn IPMC 112 spasmoneme, (a) at rest and (b) under excitation of $2 \mathrm{~V}$ (note this amount is below the maximum applicable voltage of $2.07 \mathrm{~V})$, (c) excited under $2.5 \mathrm{~V}$, as predicted the actuator tends to curl when excited through input voltages above the maximum allowance, and therefore does not move in the perpendicular plane

The one-turn IPMC spasmoneme generated a vertical displacement of $0.61 \mathrm{~cm}$ in response to $2 \mathrm{~V}$ excitation. The lower than expected actuation is the result of voltage losses along the IPMC due to the finite electrical resistance of the IPMC. In this case the IPMC had end-to-end resistance of $6.2 \Omega$ on each side. Improvements in actuator response can be achieved by increasing electrical conductivity, for example through additionally sputter coating. In the following we discuss the application of a multi-turn spasmoneme arm as a camera positioner.

\subsection{Application of an artificial spasmoneme actuator for helical scanning}

Spiral scanning involves the movement of the camera in a helical pattern for the purpose of enhanced resolution. An example of spiral scanners is the work carried by Soper et al 2011 in the development of an ultrathin scanning-fiber endoscope for bladder-surveillance endoscopy. A micro camera can be mounted on the internal end of the artificial spasmoneme such that it rotates and rises as the spasmoneme is stimulated. Note that in this configuration only one degree of freedom (i.e. one control signal) is required to achieve a full hemispherical scan.

For this purpose we base our model on Nafion 115 based IPMC actuators which provides higher mechanical strength at the expense of lower per-voltage strain. To be suitable for scanning applications, the actuator must be capable of demonstrating at least 360 degrees of rotation at its apex, where the camera is placed. The design parameters consist of: W, width of the IPMC strip; N, number of turns of the spiral; and $\mathrm{V}$, input voltage.

We consider discrete space sets for width $\mathrm{W}=\{0.1 \mathrm{~cm}, 0.2 \mathrm{~cm}, 0.3 \mathrm{~cm}\}$ and the number of turns $\mathrm{N}=\{2,3,4\}$ of the actuator and analyse their ability in meeting the system constraints. By increasing the voltage from $0.5 \mathrm{~V}$ with steps of $10 \mathrm{mV}$ to the maximum applicable voltage in each case the tip rotation around the spiral origin is calculated and is shown in figure 19, for the 9 candidate spirals. 


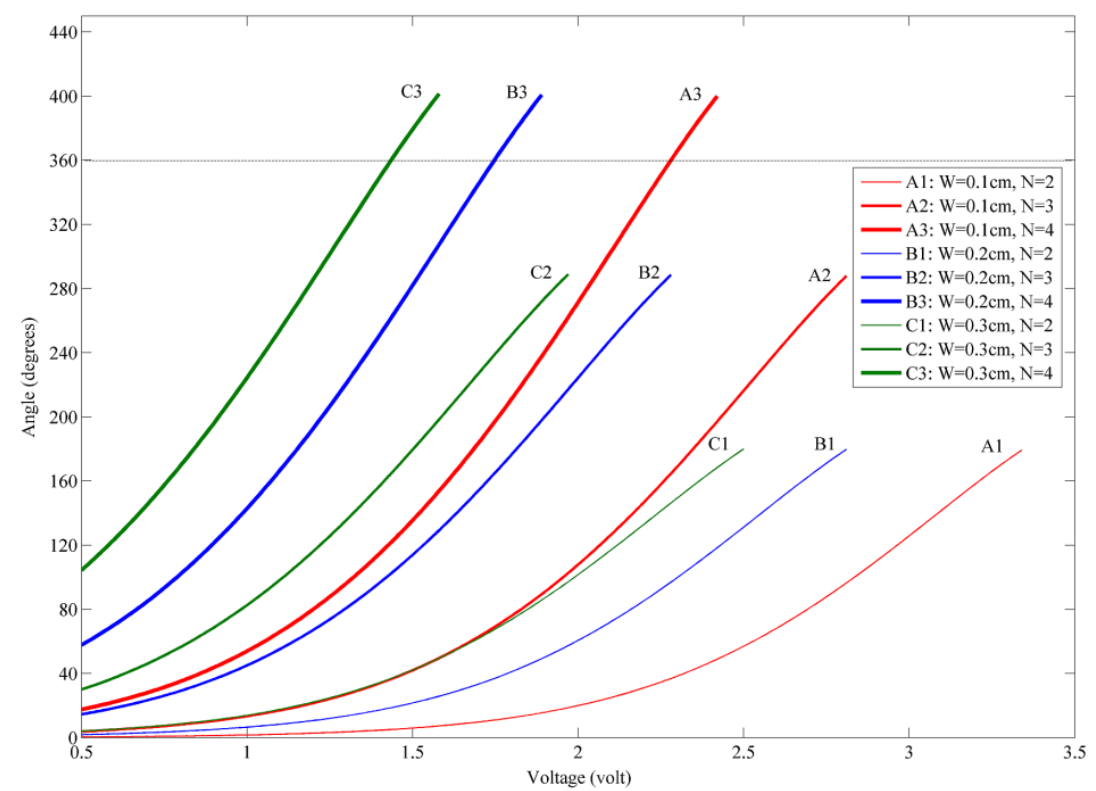

Figure 19. Tip rotation for different combinations of $\mathrm{W}$ and $\mathrm{N}$

Table3. Specification of 4-turn actuators

\begin{tabular}{|c|c|c|c|c|c|}
\hline Actuator id. & {$[\mathbf{W}(\mathbf{c m}), \mathbf{N}]$} & $V_{\text {max }}$, (volt) & $\begin{array}{c}\text { Actuator Length } \\
\text { (cm) }\end{array}$ & $\begin{array}{c}\text { Voltage for } 360^{\circ} \\
\text { (Volt) }\end{array}$ & $\begin{array}{c}\text { Rise at } V_{\text {max }} \\
\text { (cm) }\end{array}$ \\
\hline A3 & {$[0.1,4]$} & 2.43 & 6.30 & 2.28 & 2.89 \\
\hline B3 & {$[0.2,4]$} & 1.89 & 12.60 & 1.75 & 5.78 \\
\hline $\mathrm{C3}$ & {$[0.3,4]$} & 1.58 & 18.90 & 1.44 & 8.67 \\
\hline
\end{tabular}

Figure 19 shows that we can have a maximum tip rotation of 180 degrees with a 2-turn spiral actuator, however this increases to 289 and 401 degrees for $\mathrm{N}=3$ and $\mathrm{N}=4$ spirals respectively with the same width. To be suited for scanning applications, we need 360 degrees of tip rotation, and this is only achievable for a spiral actuator with at least 4 turns.

Changing the width of the IPMC strip does not affect the amount of rotation; however it changes the length of the actuator and hence changes the required excitement voltage. A 4-turn spiral actuator with $\mathrm{W}=0.3 \mathrm{~cm}$ rotates 360 degrees at $1.44 \mathrm{v}$, as summarised in Table 3 , where the required voltages for the same rotations are $1.75 \mathrm{v}$ and $2.28 \mathrm{v}$ for actuators with $\mathrm{W}=0.2 \mathrm{~cm}$ and $\mathrm{W}=0.1 \mathrm{~cm}$ respectively. Hence, the spiral actuator labelled C3 in figure 19 with four turns and $0.3 \mathrm{~cm}$ IPMC width is the ideal candidate for the realisation of camera positioner. Two and three dimensional views of the simulated $\mathrm{C} 3$ actuator in presented in figure 20. Note that the spiral has a maximum radius of $1.35 \mathrm{~cm}$. 


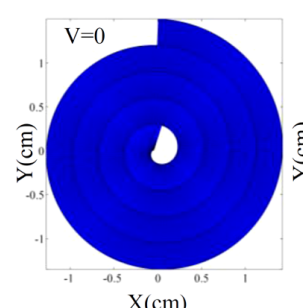

$\mathrm{X}(\mathrm{cm})$

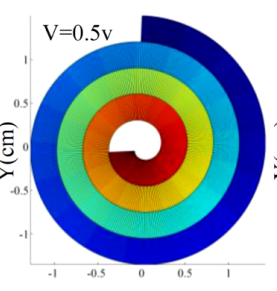

$\mathrm{X}(\mathrm{cm})$

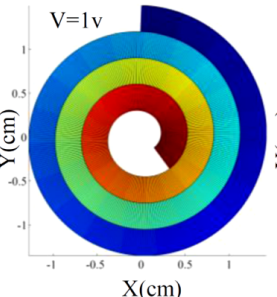

$\mathrm{X}(\mathrm{cm})$

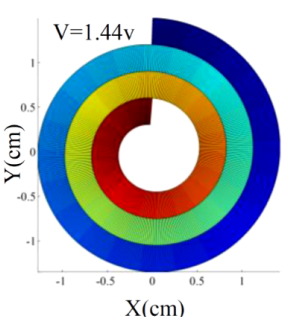

$\mathrm{X}(\mathrm{cm})$

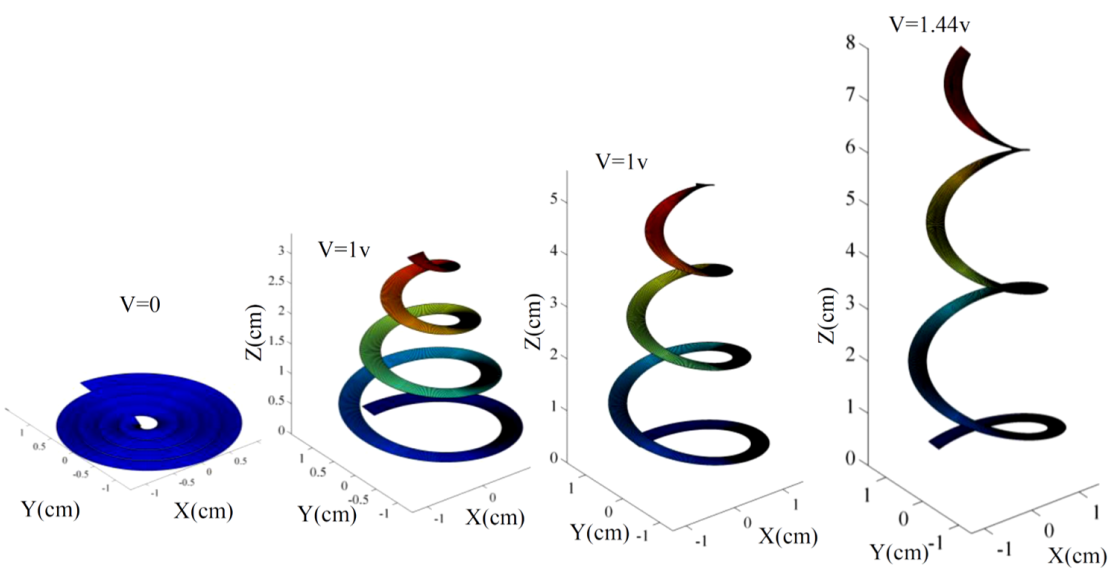

Figure 20. Two and three dimensional view of the simulated spiral actuator.

Here we have shown the spiral actuating where the outer most end is fixed. The alternative is to fix the inner-most end and allow the outer spiral to move. This alternative configuration is suitable for use in other applications, but it should be noted that during actuation it will generate higher rotational momentum and this must also be taken into account.

\section{Conclusions}

In this paper we have presented two biologically inspired artificial actuator structures. We employ kirigami design principles to turn flat IPMC membranes into 3D active structures. We have taken inspiration from the cuspid valve and Tubastrea to develop the burstbot, a fluid control and pumping structure. We have shown that the burstbot can generate asymmetric flow through an aperture in response to an asymmetric charge-balanced control signal. We have also taken inspiration from the vorticella protozoa to develop an artificial spasmoneme, termed the vortibot. The design of the spasmoneme and initial experiments show how actuation of an Achremedean spiral made from IPMC material generates a 3D conical normal helix. We have also shown how the proposed artificial spasmoneme model can be used as a camera positioner in spiral scan technologies where a single input signal translates into a hemispherical scanning path.

\section{Acknowledgements}

This research was funded by research grant EP/I032533/1 from the UK Engineering and Physical Sciences Research Council (EPSRC). 


\section{References}

Badalucco, L. (2000) Kirigami: The Art of 3-dimensional Paper Cutting, Sterling Publishing Company, 160 pages.

Bar-Cohen, Y., Kim K.J., Choi H.R., Madden J.D.W. (2007) Electroactive Polymer Materials, Smart Materials and Structures, 16:2.

Blomquist, C.H., Lima, P.H., Tarrant, A.M., Atkinson, M.J. and Atkinson, S. (2006) 173 Hydroxysteroid dehydrogenase (17ß-HSD) in scleractinian corals and zooxanthellae, Comparative Biochemistry and Physiology, Part B 143: 397-403.

France, D.C. (2007) Structure and mechanics of spasemonome, a biological spring within the protozoan vorticella convallaria, $\mathrm{PhD}$ thesis, Massachusetts Institute of Technology.

Ilievski, F., Mazzeo, A.D., Shepherd, R.F., Chen, X., Whitesides, G.M. (2011) Soft Robotics for Chemists Angewandte Chemie International Edition, Volume 50, Issue 8, pages 1890-1895.

Jeong, K.U., Jang, J.H., Jin, K.Y., Kim, Y.J., Nah, C., Lee, J.H., Lee, M.H., Wang, C.L., Cheng, S.Z.D., Thomas, E.L. (2011) Three-dimensional actuators transformed from programmed twodimensional structures via bending, twisting, and folding mechanisms, Journal of Materials Chemistry, 21 (19), 6824-6830.

Kier, W. M. and A. M. Smith (1990) The Morphology and Mechanics of Octopus Suckers, The Biological Bulletin, 178, pp. 126-136.

Kim, K., Tadokoro, S. (2007) [Electroactive polymers for robotic applications], Springer, 1st Edition, $281 \mathrm{p}$.

Mahadevan, L. and Matsudaira, P. (2000) Motility powered by supramolecular springs and ratchets. Science 288, 95-99.

Mane, P., Mossi, K. and Bryant, R. (2005). Synthetic jetswith piezoelectric diaphragms, Smart Structures and Materials, Damping and Isolation, Proceedings of the SPIE, 5761: 233-243.

Moriyama, Y., Hiyama, S., and Asai, H. (1998) High-speed video cinematographic demonstration of stalk and zooid contraction of Vorticella convallaria. Biophysical Journal 74: 487-491.

Napadow, V. J., Kamm, R. D. and Gilbert, R. J. (2002) A biomechanical model of sagittal tongue bending, Journal of Biomechanical Engineering, 124, pp. 547-556.

Saito, K., Agnese, F., Scarpa, F. (2011) Cellular Kirigami Morphing Wingbox Concept, Journal of Intelligent Material Systems and Structures, DOI: 10.1177/1045389X11416030

Sareh S., Conn, A.T., Rossiter, J.M. (2010) Optimization of bio-inspired multi-segment IPMC cilia, Proceedings of Electroactive Polymer Actuators and Devices (EAPAD XII), SPIE Vol. 7642.

Satir, P. (1983). [Cilia and related organelles], Carolina Biological Supply Company, Burlington, North Carolina, 3-9

Sanan, S., Moidel, J., Atkeson C.G. (2011) A Continuum Approach to Safe Robots for Physical Human Interaction, Proc. of 2011 International Symposium on Quality of Life Technology (isQoLT).

Shahinpoor, M. and Kim, K. J. (2005). Ionic polymer-metal composites: IV. Industrial and medical applications, Smart Materials and Structures, 14, 197-214.

Silflow, C.D., Lefebvre, P.A. (2001) Assembly and motility of eukaryotic cilia and flagella. Lessons from Chlamydomonas reinhardtii. Plant Physiol. 127(4):1500-7.

Soper, T., Seibel, E. and Porter, M. (2011) New approaches to bladder-surveillance endoscopy, 7 April 2011, SPIE Newsroom. 
Upadhyaya, A., Baraban, M., Wong, J., Matsudaira, P., van Oudenaarden, A. and Mahadevan, L. (2008) Power-limitedcontraction dynamics of Vorticella convallaria: Anultrafast biological spring, Biophysical Journal, vol.94, pp. 265-272.

Wainwright, P.C., Bennett, A. (1992) The mechanism of tongue projection in chameleons, II role of shape change in a muscular hydrostat, J. exp. Biol. 168, 23-40.

Wu, Y. and Nemat-Nasser, S. (2004) Verification of micromechanical models of actuation of ionic polymer-metal composites (IPMCs), Smart Structures and Materials 2: Electroactive Polymer Actuators and Devices (EAPAD). Edited by Bar-Cohen, Yoseph. Proceedings of the SPIE, Volume 5385, pp. 155-158. 